\title{
Fast method of speckle suppression for reflection phase microscopy
}

\author{
Patrycjusz Stremplewski ${ }^{1}$, Maciej Nowakowski $^{2}$, Dawid Borycki ${ }^{1}$, and Maciej Wojtkowski ${ }^{1,2}$ \\ ${ }^{1}$ Institute of Physical Chemistry, Polish Academy of Sciences, Kasprzaka 44/52, 01-224 Warsaw, Poland \\ ${ }^{2}$ Institute of Physics, Nicolaus Copernicus University, Grudziadzka 5, 87-100 Torun, Poland
}

Received September 17, 2018; accepted December 11, 2018; published December 31, 2018

\begin{abstract}
Light propagating in a turbid medium is randomly altered by optical inhomogeneities, which not only change the momentum and polarization of light but also generate a speckle pattern. All these effects strongly limit the capabilities of laser based, quantitative phasesensitive optical biomedical imaging modalities by hindering the reconstruction of phase distribution. Here we introduce the method of rapid incident light modulation, which allows to suppress speckle noise and preserve spatial phase distribution. We implement this approach in the full-field Michelson interferometer, where incident light is modulated using a digital micromirror device (DMD).
\end{abstract}

Phase microscopy provides high resolution 3D imaging capability, without using exogeneous contrast agents or high power excitation [1]. Narrow band light sources allow for optical design flexibility. On the other hand, long coherence time disables axial sectioning. Useful information about the object is often disturbed by multiply scattering or speckle noise from the embedding medium. Axial sectioning of the system can be restored by using dynamic speckle illumination [2-4].

Fast and straightforward methods utilize moving [5-7], rotating [8-9] or vibrating random [10] or deterministic diffusers. Diffractive optical elements [11] or resonant scanner [12] can be also used for that purpose. The efficiency of these methods strongly depends on the number $N$ of statistically independent optical fields to be averaged within the detector integration time $\Delta T$. Usually the speckle suppression of $N^{1 / 2}$ is obtained [13], but at the expense of reduced phase information.

The digital mirror device (DMD) is an example of fast spatial light modulator. A DMD is used here to implement wavefront modulation with deterministic phase patterns, derived from Hadamard matrix. These patterns were proven to provide a mutually orthogonal set of phase patterns [11].

The idea behind our method is similar to dynamic speckle illumination phase microscopy, which was experimentally presented in [14-15] and theoretically explained in [16]. In these contributions, light emerging from the laser illuminates the rotating diffuser. The resulting speckle field is split into sample and reference paths. Both fields are combined at the detector plane and produce an interference pattern. When imaging the sample, interference occurs only between the light field coming from the layer of the sample, for which focusing of the back reflected speckle pattern is the same as for the reference arm. Fields scattered from other layers are suppressed. A DMD allows for creation of a controlled intensity image refreshed at a rate of $16 \mathrm{kHz}$. By using techniques known from digital holography one can turn this intensity modulator into a phase-only light modulator. The idea is to display a diffraction grating on a DMD with locally shifted grooves, and to use diffracted light as a source of phase-modulated wave (Figs. 1a $\div \mathrm{b}$ ). A DMD is a set of micromirrors. Each micromirror is a square with the side of $13.68 \mu \mathrm{m}$, which can be rotated along its diagonal by $+12 \mathrm{deg}$. or $-12 \mathrm{deg}$. When all the pixels are $\mathrm{ON}$ or OFF, like in Fig. 1c, A DMD acts as a grating of the constant of $13.68 / \sqrt{ } 2 \mu \mathrm{m}$. In order to gain control over the wavefront, we have to imprint a more sparse grating on a DMD. The densest grating, allowing for creation of the binary $0 \div \pi$ phase-patterns is presented in Fig. $1 \mathrm{~d}$. Micromirror columns are alternately tilted by $+12 \mathrm{deg}$. and $-12 \mathrm{deg}$. For proper choice of the incident angle of the beam on the modulator surface, we regarded a DMD as a blazed diffraction grating.
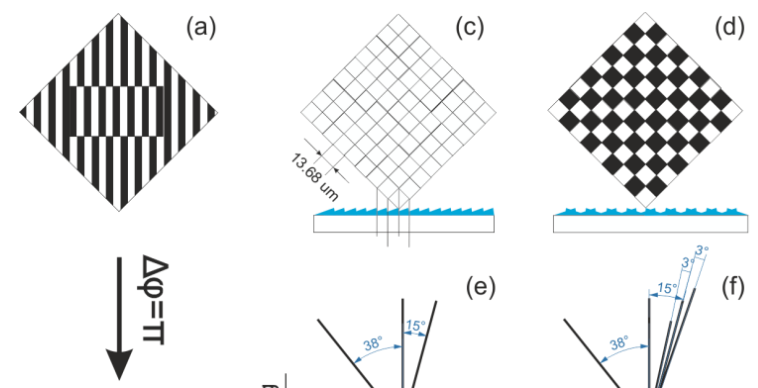

(b)
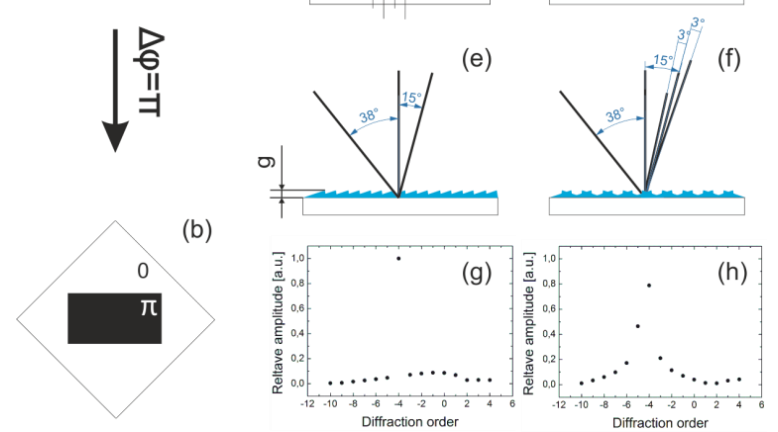

Fig. 1. DMD working as diffraction grating: $(a, b)$ diffraction grating with locally shifted groves and resulting phase pattern, (c) all micromirrors at the same state (on or off), (d) every second column on the most dense controllable grating, (e, f) angles of diffraction on 
gratings (c) and (d), (g, h) calculated relative intensities of light diffracted to different orders by the grating (a) when the angle of incidence of the input beam is $38 \mathrm{deg}$. and common choice of $24 \mathrm{deg}$.

We found, that the angle of incidence of about $38 \mathrm{deg}$. ensures minimization of the intensity of light diffracted into other beams (compare Fig. 1g and Fig. 1h).

Imaging is performed with a Michelson interferometer. The phase is controlled with a piezoelectric transducer (PZT) (Fig. 2a). The collimated beam of an 880nm laser diode (LD) homogeneously illuminates the active area of a DMD.

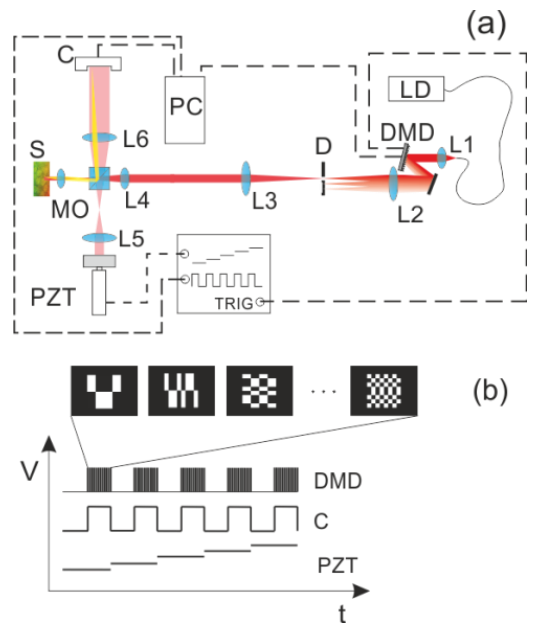

Fig. 2. (a) Experimental setup, (b) acquisition and phase modulation synchronization diagram.

The beam reflected from the grating imprinted on a DMD is diffracted into consecutive orders. We pick the 1st diffraction order, and block the other ones with a diaphragm $(D)$. Lens L4 $(f=100 \mathrm{~mm})$ and microscope objective MO $(20 \times$ Olympus, NA=0.4) form another $4 \mathrm{f}$ system in order to ensure homogeneous sample illumination.The reference and sample waves are split and combined at a non-polarizing beam splitter. Lens L6 $(f=150 \mathrm{~mm})$ acts as a tube lens, and it comprises $4 f$ configurations with both the microscope objective and L5. Images are acquired with a CMOS camera. The triggering signal for the camera and driving voltage for PZT come from a 2 channel function generator. A DMD controller sends a synchronization signal each time it starts displaying a defined sequence of images. This signal serves as a trigger for a function generator. We used a set of $N=1024$ Hadamard masks, and all this sequence of phase masks was repeated $M=5$ times, one time for each reference mirror position and single camera acquisition (Fig. 4b). Consecutive positions of the reference mirror are separated by $110 \mathrm{~nm}$ to shift the phase of reference radiation by 90 degrees between subsequent acquisitions. Phase patterns were imprinted on a DMD at a rate of 10 000 masks/s, thus all the procedure of data acquisition took about half a second. Each data set comprises 5 images.

In order to retrieve phase information, we used traditional 5-step phase microscopy. This allows also for using a simple Michelson interferometer and imaging in reflection mode without losing half of the signal, like in $[6,14]$.

The method was first applied to image a 1951 USAF resolution test chart, covered by a diffuser $(30-\mu \mathrm{m}$-thick layer of $1-\mu \mathrm{m}$-diameter aluminum oxide balls of optical thickness of 8 mean free paths - estimated with a transmission set-up, like in [17]). We collected images of the same object with and without phase modulation.

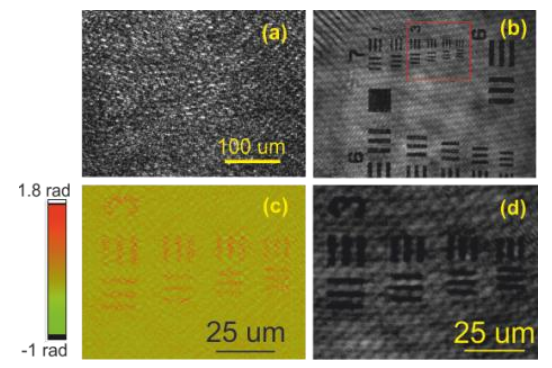

Fig. 3. Imaging of a 1951 USAF test target. (a, b) Intensity images retrieved from interferograms without and with wavefront shaping, (c, d) phase and intensity images of the area marked in (b) with a red rectangle.

When no modulation is applied, the intensity image (reconstructed from the set of five interferograms) of the USAF pattern contains speckle noise, which hinders bars and labels of the resolution chart (Fig. 3a). Intensity images obtained with modulation $\mathrm{ON}$ are depicted in Fig. $3 \mathrm{~b}$ and Fig. 3d clearly show USAF features. The phase image (Fig. 3c) was also utilized to estimate the thickness of the reflecting layer of the test target. We obtained the thickness of about $100 \mathrm{~nm}$, which is close to that declared by the producer value of $120 \mathrm{~nm}$. Similarly as in [14], spatially incoherent illumination allows for improving the resolution of the set-up. In our case, the resolution for coherent illumination is $1.22 \lambda / \mathrm{NA}=2.7 \mu \mathrm{m}$, but we can clearly distinguish USAF test target bars separated by $2.2 \mu \mathrm{m}$ (6-th element of the 7-th group, Fig. 3d), when wavefront modulation is on, even when imaging through the diffuser. The results of further tests on resolution are presented in Fig. 4. Without a diffuser we estimate the theoretical resolution for incoherent illumination of $1.35 \mu \mathrm{m}$. Line spread function (LSF) (Fig. 4b) calculated from the measured edge response (Fig. 4a) by differentiation [18] is of about $1.4 \mu \mathrm{m}$ and bars of the USAF test target are distinguishable up to 5-th element of 8 -th group (Fig. $4 \mathrm{c}$ and $4 \mathrm{~d}$ ), which are $1.23 \mu \mathrm{m}$ wide. 

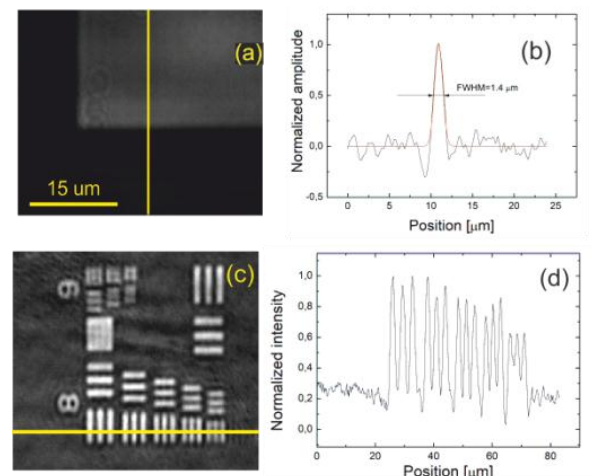

Fig. 4. Quantitative analysis of the spatial resolution. (a) Image of the edge of the metal square on the USAF test target with yellow line indicatingthe region of interest (ROI) used for further analysis, (b) LSF obtained by differentiation of ROI pixel intensities, FWHM of fitted Gaussian function (red curve) is $1.4 \mu \mathrm{m},(\mathrm{c}, \mathrm{d})$ image of 8 -th group of USAF test target and pixel intensity along horizontal yellow line in (c).

Finally, we present set-up performance to improve imaging of the $40 \mu \mathrm{m}$ thick cat brain slice ex vivo (Fig. 5a). During slice preparation neuronal nuclei were stained with silver. Phase-modulated images were compared with white light illumination (halogen lamp) in transmission mode. The latter was incorporated to the set-up from Fig. $2 \mathrm{a}$ via a multimode fiber bundle, which end was placed below the sample. The intensity image obtained with broad spectrum illumination is presented in Fig. 5c. There are barely recognizable silhouettes of neuronal nuclei, and the contrast of the image is very low. We can compare this result to the intensity image retrieved from the set of interferograms with wavefront modulation (Fig. 5d), where we clearly see the locations of neuronal nuclei. Again, we can obtain a phase map (Fig. 5b), which correlates well with intensity images obtained with spatially incoherent (Fig. 5c) and coherent light sources (Fig. 5d), though both Fig. 5b and Fig. 5d still suffer from some phase or intensity instabilities, pronounced as sinusoidal horizontal and oblique modulation.
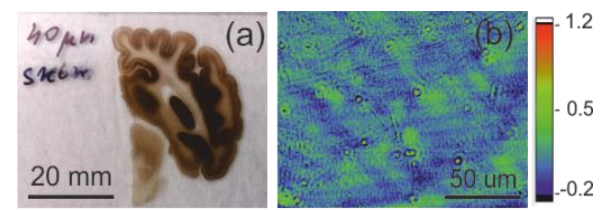

(c)

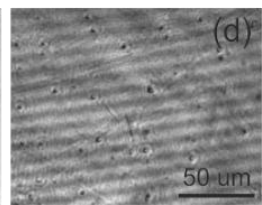

Fig. 5. (a) photograph of cat brain slice, (b, d) phase contrast and intensity images retrieved from interferograms with wavefront manipulation method, (c) intensity image obtained with white light illumination in transmission mode.
In this paper we presented a fast method for suppressing multiple scattering noise in phase-sensitive optical coherence microscopy. Our technique allows for acquiring full data sets at a rate of $2 \mathrm{~Hz}$ with the number of phase patterns of 1024. Importantly, deterministic phase manipulation allows to suppress the noise without blurring the image and losing phase information. Therefore, this approach can be also used to image phase objects. Fast and deterministic control over the wavefront of an illumination beam might be helpful for optimization of random speckle illumination methods. The contrast of intensity images appeared superior to that obtained for white light illumination. The presented DMD-based modulation enables to deliver to the object only $6 \%$ of the power of the laser, which is acceptable for microscopic applications, but for any imaging which requires high power on the sample, more efficient modulators should be used.

This work was funded by the National Science Center (NCN) 2016/22/A/ST2/00313. MW acknowledges funding from the European Union's Horizon 2020 research and innovation program under grant agreement No 666295. We are grateful Grzegorz Wilczyński for providing rat skin samples.

\section{References}

[1] F. Zernike, Physica 9, 974 (1942).

[2] M.C. Pitter, C.W. See, M.G. Somekh, Opt. Lett. 29, 1200 (2004).

[3] N.B.E. Sawyer, S.P. Morgan, M.G. Somekh, C.W. See, X.F. Cao, B.Y. Shekunov, E. Astrakharchik, Rev. Scient. Instrum. 72, 3793 (2001).

[4] G.W. John, I.H. Keith, Measur. Scien. Technol. 24, 125404 (2013).

[5] S. Lowenthal, D. Joyeux, J. Opt. Soc. Am. 61, 847 (1971).

[6] S. Kubota, J.W. Goodman, Appl. Opt. 49, 4385 (2010).

[7] Y. Li, H. Lee, E. Wolf, J. Modern Opt. 52, 791 (2005).

[8] C.-Y. Chen, W.-C. Su, C.-H. Lin, M.-D. Ke, Q.-L. Deng, K.Y. Chiu, Opt. Rev. 19, 440 (2012).

[9] J. Lehtolahti, M. Kuittinen, J. Turunen, J. Tervo, Opt. Expr. 23, 10453 (2015).

[10] J.-W. Pan, C.-H. Shih, Opt. Expr. 22, 6464 (2014).

[11] J.I. Trisnadi, Opt. Lett. 29, 11 (2004).

[12] M. Szkulmowski, I. Gorczynska, D. Szlag, M. Sylwestrzak, A. Kowalczyk, M. Wojtkowski, Opt. Expr. 20, 1337 (2012).

[13] J.W. Goodman, Speckle phenomena in optics: theory and applications (Roberts and Company Publishers, 2006).

[14] Y. Choi, P. Hosseini, W. Choi, R.R. Dasari, P.T.C. So, Z. Yaqoob, Opt. Lett. 39, 6062 (2014)

[15] Y. Choi, T.D. Yang, K.J. Lee, W. Choi, Opt. Lett. 36, 2465 (2011).

[16] R. Zhou, D. Jin, P. Hosseini, V.R. Singh, Y.-H. Kim, C. Kuang, R.R. Dasari, Z. Yaqoob, P.T.C. So, Opt. Expr. 25, 130 (2017).

[17] M. Schmitz, T. Rothe, A. Kienle, Biomedical Opt. Expr. 2, 2665 (2011).

[18] P. Judy, The line spread function and modulation transfer function of a computer tomography scanner, vol. 3, pp. 233-236 (1976), 
